\title{
Editorial to the Special Issue on Reconstruction of Porous Media and Materials and Its Applications
}

\author{
Pejman Tahmasebi ${ }^{1} \cdot$ Muhammad Sahimi $^{2}$
}

Published online: 10 August 2018

(c) Springer Nature B.V. 2018

Disordered multiphase porous media, ranging from rock, membranes, adsorbents, asphalt, wood, and concrete, to biological tissues, are ubiquitous. Their understanding is of fundamental importance, and their accurate characterization and modeling are critical to various branches of science and engineering. In most cases, however, one must confront the fact that experimental data and insights into the structure of porous media are limited. Thus, methods that can use limited information and construct models of porous media in order to assess their properties and study various phenomena in them are most cost-effective. Class approaches that have been developed over the past three decades to address the problem is based on what is usually referred to reconstruction: given a certain amount of data for a given heterogeneous porous medium, how can one develop a model for the medium that not only honors the data, but also provides accurate predictions for those properties of the medium for which no data are available, or are hard to measure, or were not used in the reconstruction?

Thus, the class of such methods is quite broad, ranging from process-based approaches-those that try to mimic the processes that produce porous media of interest-and object-based techniques-those that treat the pore and grain structures as a set of objects that are defined based on a prior knowledge of the pore space-to statistical methods in which one tries to use various two-point spatial correlation functions to extract the maximum amount of information from images that are used to reconstruct the porous media, and combines them with an optimization algorithm, e.g., simulated annealing and the genetic algorithm. The research field of reconstruction has been very active and is still witnessing significant developments, due to the recent understanding of complex physics of porous media revealed by advanced imagining technologies. Further development of such methods still requires incorporating deep physical concepts and complexities of disordered media, which are expected to play a significant role in the future studies of all types of porous structures. Some examples are shown below.

Pejman Tahmasebi ptahmase@uwyo.edu

Muhammad Sahimi moe@usc.edu

1 Department of Petroleum Engineering, University of Wyoming, Laramie, USA

2 Mork Family Department of Chemical Engineering and Materials Science, University of Southern California, Los Angeles, USA 


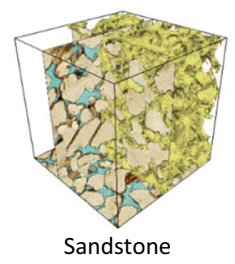

Sandstone

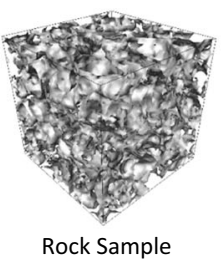

Rock Sample

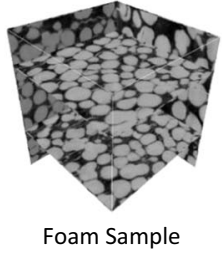

Foam Sample
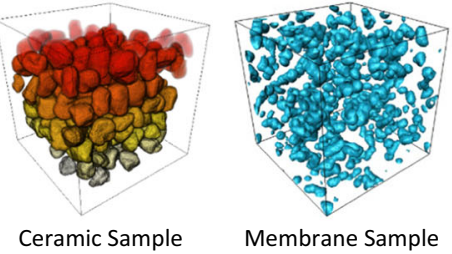

This special is devoted to the recent developments in the reconstruction approaches. The goal of the issue is to cover the most recent theoretical and computational progress in the field and to bring together the development of advanced algorithms, as well as practical applications of reconstruction methods to all types of porous media.

Seven papers are included in this special issue, containing various problems and reconstruction techniques. Given the lack of data in porous media modeling, the first paper by Li et al. presents a reconstruction method that generate several stochastic models by random fusing of limited bimodal microstructural data, such as limited-angle X-ray tomographic radiographs and 2D optical micrographs through certain lower-order correlation functions.

The paper by Naraghi et al. uses the object-based methods along with the information extracted from the thin sections in order to build several stochastic 2D models for shale sample. The authors use mineralogical information and pore-size distributions from X-ray diffraction.

The paper by Izadi et al. discusses the effect of image resolution on 3D reconstruction. The authors investigate how images with different resolutions can affect the selection of the representative elementary volume (REV) and also compare their findings with the experimental data.

The paper by Čapek compares various simulated annealing algorithms, used in reconstruction techniques, which are constrained by low-order microstructural descriptors, and controlled by four annealing schedules for reducing the "temperature." The annealing schedule includes the exponential decay, as well as three adaptive schedules that deduce the optimum cooling speed from such statistical measures as the mean "energy" of the system and its standard deviation.

The paper by Mosser et al. is focused on the application of generative adversarial neural networks in image processing, as it requires no a priori inference of the probability distribution associated with the training data. The convolutional neural network allows sampling of large and realistic volumetric images. The authors apply the method to a micro-CT dataset for oolitic Ketton limestone and compare the generated images with the actual data.

The paper by Piasecki et al. describes an approach that uses simulated annealing and the so-called entropic descriptors. For a binary pattern, they quantify spatial inhomogeneity or statistical complexity at discrete length scales. The EDs extract structural information that is not similar to what the traditional two-point correlation functions produce. The authors show that their reconstruction method is computationally efficient, if it begins with a preliminary synthetic configuration that has some desirable features, or if uses low-cost approximate reconstructing of the entire multiphase medium beyond simulated annealing technique.

The last paper by Yao et al. is about a process-based pore-network modeling of shale samples. They have implemented a process-based approach to reconstruct a multiscale network comprising of microscale, nanoscale pores in organic matter and nanoscale pores in clay aggregates. The process-based approach tries to reproduce the geological processes by considering the internal connections between the pore spaces and accounting for pore distributions in organic matter and clay elements. 
We hope that the published papers in this special issue will open new venues and developments in the field of porous media reconstruction. The papers are a combination of the previously developed methods and some new and excellent innovations that require future research. 